\title{
Control of the ST7 Disturbance Reduction System Flight Experiment
}

\author{
P. G. Maghami*, O. C. Hsu*, J. R. O’Donnell, Jr.* \\ * Mission Engineering and Systems Analysis Division, NASA Goddard Space Flight Center \\ Greenbelt, MD 20771
}

\begin{abstract}
The Space Technology 7 (ST7) experiment will perform an on-orbit system-level validation of two specific Disturbance Reduction System technologies: colloidal micronewton thrusters and drag-free control. The ST7 Disturbance Reduction System (DRS) is designed to maintain the spacecraft's position with respect to a free-floating test mass while limiting the residual accelerations of that test mass over the frequency range of 1 to $30 \mathrm{mHz}$. This paper presents the overall design and analysis of the spacecraft drag-free and attitude controllers, with particular attention given to its primary mission mode. These controllers close the loop between the drag-free sensors and the colloidal micronewton thrusters.
\end{abstract}

Keywords: Drag-Free, Disturbance Rejection, Colloidal Micronewton thruster, Drag-Free Sensor PACS: $04.80 . \mathrm{Nn}, 95.55 . \mathrm{Ym}$

\section{INTRODUCTION}

The Space Technology 7 (ST7) mission is a Disturbance Reduction System (DRS) flight validation experiment within NASA's New Millennium Program [1]. The ST7 DRS is tasked to validate two specific technologies: drag-free control and colloidal micronewton thrusters (CMNT) to provide low-noise control of the spacecraft for dragfree flight. This validation is performed using highly sensitive drag-free sensors (DFS), which is provided by the LISA Technology Package (LTP) of the European Space Agency (ESA), to measure and/or actuate the position and attitude of a spacecraft with respect to an internal free-floating test mass. The ST7-DRS is designed to maintain the spacecraft's position, with respect to the DFS' free-floating test mass, to less than 10 $\mathrm{nm} / \sqrt{\mathrm{Hz}}$, over ST7's science measurement frequency range from 1 to $30 \mathrm{mHz}$ [2]. This requirement will help ensure that the residual accelerations on the test masses (beyond gravitational acceleration) will be below the ST7 goal of $3 \times 10^{-14}\left(1+[\mathrm{f} / 3 \mathrm{mHz}]^{2}\right)$ $\mathrm{m} / \mathrm{s}^{2} / \sqrt{\mathrm{Hz}}$ [2]. The current operational baseline is such that the Lisa Pathfinder spacecraft would handover the control of the spacecraft to the DRS at specific times so that it may conduct its experiments. This paper presents the overall design of ST7-DRS control system that closes the loop between the DFS units and the CMNT. However, its main emphasis is the control system in the dual drag-free mode, which is the primary mode of the mission. 


\section{CONTROL MODES}

Currently, there are six functional control modes envisioned in the ST7 Disturbance Reduction system. These are the Attitude Only mode, in which only the spacecraft attitude is controlled using star tracker (ST) data, and the test masses are controlled in their accelerometer modes; Zero-G mode, in which both spacecraft attitude and position are controlled using ST data as well as the acceleration signals from one of the DFS units; Drag-Free/High-Force mode, which is the same as the previous mode except relative position errors from a DFS are used to establish drag-free motion of one test mass, and the reference test mass (RTM) is controlled in rotation (in high force actuation mode) and the non-reference test mass (NTM) is in accelerometer mode; Drag-Free/Low-Force mode, which is the same as the previous mode except the RTM is controlled in rotation using the low force actuation mode; 18 degree of freedom (DOF) Transitional mode, which is the same as the previous mode except the NTM is now controlled in the low force actuation mode; and finally the 18-DOF mode, in which both test masses are in drag-free motion in the measurement band. The first five modes are used to transition the spacecraft to the science mode, which is the primary mode of the mission. The control design for this mode is detailed in the following.

\section{CONTROL DESIGN}

\section{Attitude Control}

The attitude control system (ACS) uses the spacecraft attitude error, which is generated by computing the difference between the quaternion provided by the star tracker and the target quaternion, to generate torque commands (implemented through CMNT). In the 18-DOF mode, the ACS is designed to be a very low bandwidth controller for two reasons. First is to allow the drag-free control system to use spacecraft torque authority in the measurement bandwidth (MBW) for drag-free operations, hence the ACS must attenuate well below the start of the MBW. The measurement bandwidth is between 1 and $30 \mathrm{mHz}$. Second is to avoid star tracker noise bleeding into the MBW and beyond, hence resulting in excessive accelerations on the test masses. Of course, the bandwidth has to be high enough to meet the requirements on spacecraft pointing and rate. The ACS design is a single-input/single output (SISO) controller with each loop comprised of a Proportional-IntegralDerivative (PID) controller with a $6^{\text {th }}$ order attenuation filter.

$$
T(s)=K_{a l}(s) \theta(s)
$$

Here, controller $K_{a l}(s)$ denotes the low-bandwidth attitude control designed to maintain the coarse pointing requirements of the spacecraft, $\theta(s)$ represents the attitude 
error vector, and the vector $T(s)$ represents the spacecraft control torque command in spacecraft body frame (B frame).

\section{Drag-Free Control System}

The drag-free control system (DFCS) is tasked with maintaining drag-free motion of six degrees of freedom in the MBW. The six degrees of freedom are the displacements of the RTM, the roll attitude of the RTM, and the transverse displacements of the nonreference test mass. The drag-free controller (DFC) and the Complementary Attitude Controller (CAC) comprise the DFCS.

\section{Drag-Free Controller}

The DFC controls the translations of the spacecraft to follow the RTM. Note that either test mass can be the reference test mass. The bandwidth of the DFC is significantly larger than the ACS to provide adequate disturbance rejection in the MBW. The main disturbance is the thruster force noise. Because of the high bandwidth of the DFC, there is no concern for any adverse couplings from the ACS. For the same reason, there is no need for any additional accommodation of the unstable poles of the plant, which may come about because of the total negative stiffness effects of electrostatic and/or self-gravity. Nevertheless, the stiffness effects are included in the analysis of the overall DRS control, which includes the DFC. The stiffness values assumed for the analysis is obtained from the DFACS requirements document [3]. For both test masses, a negative definite matrix is assumed for the total stiffness matrix, to be conservative. Because the stiffness matrices are diagonally dominant, only diagonal elements of the matrix are used in the control design and stability analysis. However, the full matrix is used in performance analysis, e.g., estimation of residual accelerations on the test masses. The DFC control command is computed as follows.

$$
F(s)=K_{D F C}(s) A \delta_{r}(s)+F_{f f}(s)
$$

Where $K_{D F C}(s)$ denotes the drag-free controller, matrix $A$ denotes the coordinate transformation matrix from test mass frame to the spacecraft body frame, the vector $\delta_{r}$ represents the spacecraft position error about the RTM, vector $F(s)$ represents spacecraft control force command in the B frame, and vector $F_{f f}(z)$ represents a feedforward command based on the current total spacecraft control torque command. This vector is computed from

$$
F_{f f}(s)=M_{S C} \vec{p}_{R T M} \times\left[\dot{J}_{S C}^{-1} T_{c}(s)\right]
$$

Where $M_{S C}$ denotes the mass of the spacecraft, vectors $\vec{p}_{R T M}$ represents the vector from the spacecraft center of mass to the nominal position of the RTM, matrix $J_{S C}$ denotes a diagonal matrix whose elements are the diagonal elements of the inertia 
matrix of the spacecraft, and vector $T_{c}(s)$ represents the current spacecraft torque command issued by the combined ACS and CAC. This command decouples the spacecraft rotation and translation control loops. The DFC design is a SISO controller, which includes lead and lag compensators, integral action, shaping filters, and a $4^{\text {th }}$ order attenuation filter.

\section{Complementary Attitude Controller}

The complementary attitude controller is responsible for establishing drag-free motion in three degrees of freedom in the MBW. These degrees of freedom are the transverse translational degrees of freedom ( $\mathrm{y}$ and $\mathrm{z}$ axes) of the NTM, and the relative roll attitude (about X-axis) of the RTM. The CAC loop is a 5-input/3-output controller, which uses the relative roll attitude error of the RTM, and the relative position errors of the test masses in the transverse directions to compute the required spacecraft torque commands.

$$
T_{a h}(s)=K_{a h}(s)\left\{\begin{array}{l}
\theta_{r x}(s) \\
\delta_{r y}(s) \\
\delta_{r z}(s) \\
\delta_{n y}(s) \\
\delta_{n z}(s)
\end{array}\right\}
$$

$K_{a h}(s)$ represents the $\mathrm{CAC}$, and is a series combination of lead-lag filter, shaping filter, and an attenuation filter; $\theta_{r x}(s)$ denotes the relative roll attitude error of the RTM; $\delta_{r y}(s)$ and $\delta_{r z}(s)$ denote the y and $z$ displacements of the test mass no.1; $\delta_{n y}(s)$ and $\delta_{n z}(s)$ represent the $\mathrm{y}$ and $\mathrm{z}$ displacements of test mass no. 2 ; and $T_{a h}(s)$ denotes the $\mathrm{CAC}$ control torques in the spacecraft $\mathrm{B}$ frame. The relative position error of the test masses in the transverse direction are used to compute the attitude error (in DFS frame) of the spacecraft about the RTM along the transverse axes ( $y$ and $z$ ). The roll attitude error of the RTM is used as the spacecraft attitude error along the roll of the DFS frame. The required rotation command is then transformed into the $\mathrm{B}$ frame and is used as an input to a SISO-based controller to regulate the spacecraft attitude in the science band. To obtain a pure rotation about the RTM, feed-forward translation commands are generated and issued to the drag-free controller. The spacecraft orientation error (in the B frame) is computed from the required rotations about the RTM in the LTP frame.

$$
\begin{aligned}
& \theta_{x}^{\prime}=\theta_{r x} \\
& \theta_{y}^{\prime}=\left(\delta_{z 1}-\delta_{z 2}\right) / \ell \\
& \theta_{z}^{\prime}=\left(-\delta_{y 1}+\delta_{y 2}\right) / \ell
\end{aligned}
$$

And the spacecraft attitude error (in the B frame) is written as 


$$
\left\{\begin{array}{l}
\theta_{x} \\
\theta_{y} \\
\theta_{z}
\end{array}\right\}=A\left\{\begin{array}{l}
\theta_{x}^{\prime} \\
\theta_{y}^{\prime} \\
\theta_{z}^{\prime}
\end{array}\right\}
$$

Where $\delta_{y 1}(s)$ and $\delta_{z 1}(s)$ denote the $y$ and $z$ displacements of the test mass no. $1 ; \delta_{y 2}(s)$ and $\delta_{z 2}(s)$ represent the $y$ and $z$ displacements of test mass no. $2 ; \ell$ denotes the nominal distance between the test masses; and $A$ represents the attitude matrix from the LTP frame to the B frame.

\section{Suspension Control}

\section{Reference Test Mass}

As the three relative translation degrees of freedom of the RTM are controlled by the $\mathrm{DFC}$, there is only suspension control of the relative attitude of the test mass with respect to its housing. The controller design is based on a SISO approach. The controller bandwidth for each axis is selected to stabilize a potentially unstable plant due to total stiffness effects, provide sufficient disturbance rejection for the test mass relative attitude, and ensure that suspension actuation cross-talk does not impose excessive accelerations on the RTM in the sensitive axis. The roll axis control is benefited by the $\mathrm{CAC}$ in providing drag-free motion of that degree of freedom at and around the MBW. However, the attitude along the other axis solely depends on suspension control. The controllers were designed using classical methods to provide sufficient gains below $\mathrm{MBW}$ for disturbance rejection, while ensuring that the gains within band are low enough as not to cause excessive acceleration on the test mass. They use the elements of the relative attitude error vector $\theta_{r}(s)$, which is obtained from capacitive/optical measurement, to compute the required electrostatic suspension torque commands.

$$
T_{r}(s)=K_{r}(s) \theta_{r}(s)+T_{f f}(s)
$$

Here, controller $K_{r}(s)$ denotes the low-bandwidth TM attitude control designed to maintain the relative attitude of the test mass, vector $T_{r}(s)$ represents the suspension torque command in test mass frame, and vector $T_{f f}(s)$ represents a feedfoward command from the current torque command of the low bandwidth ACS controller, and is given by

$$
T_{f f}(s)=J_{T M}\left[\begin{array}{ccc}
1 & 0 & 0 \\
0 & 0 & 0 \\
0 & 0 & 0
\end{array}\right] A^{T} J_{S C}^{-1} * T_{a l}(s)
$$

Matrix $J_{T M}$ denotes the test mass inertia matrix, matrix $A$ denotes the transformation matrix from the TM frame to the spacecraft body frame, matrix $J_{S C}$ denotes a diagonal matrix whose elements are the diagonal elements of the inertia matrix of the 
spacecraft, and vector $T_{a l}(s)$ represents the current torque command issued by the ACS. The bandwidth of each controller is limited, at the high end, by the limits of control torques in the low force mode of the LTP sensor.

\section{Non-reference Test Mass}

Both relative translational and rotational aegrees of freedom of the NTM are controlled via electrostatic suspension. The test mass stiffness matrix used in the design is the diagonal subset of the matrix given in Eq. 4.1.1. The attitude control part is very similar to that for the reference test mass. The main difference is in the rollaxis. Unlike the case for the RTM, the roll-axis attitude control is solely based on electrostatic suspension control. Similarly, the controller bandwidth for each axis is selected to stabilize a potentially unstable plant due to total stiffness effects, provide sufficient disturbance rejection for the test mass relative attitude, and ensure that suspension actuation cross-coupling does not impose excessive accelerations on the test mass in the sensitive axis. Each controller uses the elements of the relative attitude error vector $\theta_{n}(s)$, which is obtained from capacitive/optical measurement, to compute the required electrostatic suspension torque commands.

$$
T_{n}(s)=K_{n r}(s) \theta(s)
$$

Here, controller $K_{n r}(s)$ denotes the NTM attitude control designed to maintain the relative attitude of the NTM, and the vector $T_{n}(s)$ represents the suspension torque command in test mass frame. The translational degrees of freedom of the NTM are controlled via electrostatic suspension below the MBW. However, in two degrees of freedom the suspension controller are aided by the $\mathrm{CAC}$, which provides drag-free control for NTM displacements about the transverse (normal to the LTP axis) directions at or around the MBW. Hence, the controller bandwidth for each axis is selected to stabilize a potentially unstable plant due to total stiffness effects, provide sufficient disturbance rejection, and ensure that suspension action does not induce excessive residual accelerations along the sensitive axis due to suspension actuation cross-talk. Each controller uses the elements of the relative displacement error (gap error) vector $\delta_{n}(s)$, which is obtained from capacitive/optical measurement, to compute the required electrostatic suspension force commands.

$$
F_{n}(s)=K_{n t}(s) \delta_{n}(s)+F_{f f}(s)
$$

Here, controller $K_{n t}(s)$ denotes the test mass suspension control designed to maintain the relative position of the NTM, vector $F_{n}(s)$ represents the suspension force command in test mass frame, and vector $F_{f f}(s)$ represents a feedforward command from the current torque command of the ACS controller. This vector is computed from

$$
F_{f f}(s)=M_{T M} A^{T}\left(\vec{p}_{R T M}-\vec{p}_{N T M}\right) \times\left[J_{S C}^{-1} T_{a l}(s)\right]
$$


Where $M_{T M}$ denotes the mass of the test mass, matrix $A$ denotes the transformation matrix from the test mass frame to the spacecraft body frame, vectors $\vec{p}_{R T M}$ and $\vec{p}_{N T M}$ represent vectors from the spacecraft center of mass to the RTM and NTM, respectively; matrix $J_{S C}$ denotes a diagonal matrix whose elements are the diagonal elements of the inertia matrix of the spacecraft, and vector $T_{a l}(s)$ represents the current torque command issued by the ACS. The most challenging part of this design is the $\mathrm{X}$-axis controller, which is solely responsible for stabilizing an unstable plant with a pole as far right as $0.225 \mathrm{mHz}$. To stabilize such a plant, the cross-over frequency has to be placed above $0.225 \mathrm{mHz}$ with some margin. However, the controller has to attenuate well before the MBW (at $1 \mathrm{mHz}$ ) in order not to impart excessive actuation-induced accelerations along the sensitive axis.

\section{ANALYSIS AND DISCUSSION}

\section{Evaluation Models}

The performance of the system was evaluated using frequency and time domain analyses. The frequency domain analysis utilized the 18-DOF control design model to generate root power spectral densities (PSD) of the performance metrics and control commands. The performance metrics include relative position and attitude of the test masses. The power spectral densities were used to assess the performance of the system within the MBW. Time domain analysis of the system was also performed. A high-fidelity model was used to generate the time histories of the performance metrics and control commands. The high fidelity model includes nonlinear kinematics and dynamics of the system, CMNT dynamic logic, true disturbance models, etc. The time histories were used to verify the peak-to-peak response of the performance metrics, CMNT thrust levels, as well as absolute suspension command levels. The time histories are also used to validate the power spectral densities of the performance metrics obtained in the 18-DOF design model.

\section{Reference Test Mass}

The relative displacement of the RTM to its housing, as well as the relative attitude of the RTM about the roll axis, comprise the drag-free coordinates of the RTM in this DRS mode. The root power spectral densities (PSD) of these coordinates, and the remaining two attitude degrees of freedom, are provided in Figure 1. The root PSD plots show the contributions from various disturbance sources. The contribution of each disturbance category represents the root sum squared (RSS) values for that category; for example, the thruster noise plot is the RSS contribution of the noise from all eight thrusters. The dashed red line indicates the required performance level. The PSD plots indicate that the requirements are handily met with margins. The root PSD plots are mainly dominated by the thruster noise and measurement noise in the MBW. It is possible to reduce the peak PSDs in the vicinity of $30 \mathrm{mHz}$ at the expense of requiring higher frequency thruster activity; the current design is a compromise. The PSD plots of relative attitude are also dominated by the CMNT noise, and to a lesser degree by the sensing noise. This is expected because of the low bandwidth of the suspension loops. 

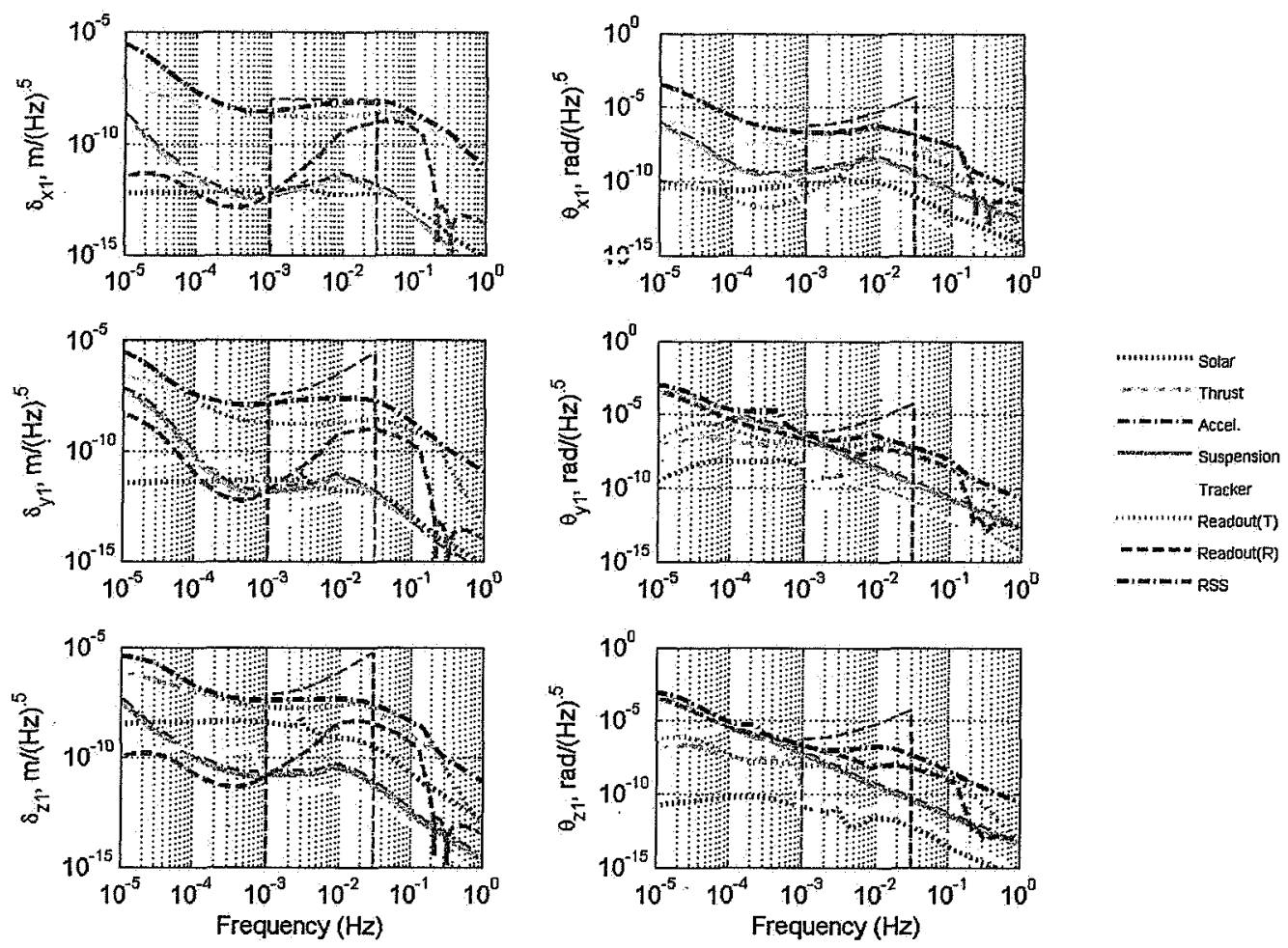

Figure 1 Root PSD Plots for the Reference Test Mass

\section{Non-Reference Test Mass}

The root PSD plots for the relative position and attitude of the NTM are shown in Figure 2. There are two drag-free coordinates for the NTM, and these are the transverse displacements of the test mass. These plots indicate that the requirements are met in all degrees of freedom. The PSD plots for the test mass displacements are mainly dominated by either the thruster noise. The relative attitude is mainly dominated by the large capacitive sensing noise, as well as the thruster noise, in all three axes.

\section{Acceleration Performance}

The overall design has a goal of achieving residual accelerations along the measurement axes of both test masses to better than $30(1+\mathrm{f} /(3 \mathrm{mHz}))^{2} \mathrm{fm} / \mathrm{s}^{2} / \mathrm{VHz}$ in the measurement band. The contributions to the accelerations come mainly from direct acceleration noise on the test mass, the electrostatic actuation induced accelerations, and test mass stiffness induced accelerations. The control design impacts only the latter two. The actuation induced accelerations are based on the RSS of the contribution from the suspension forces and torques at each frequency. Each contribution is weighted by the absolute value of the corresponding element of the total actuation cross-talk matrix. The stiffness induced accelerations are based on the 
RSS of the contribution from the three relative displacements and the three relative attitude errors at each frequency. Each contribution is weighted by the absolute value of the corresponding element of the total actuation stiffness matrix. The DFACS requirements document allocates $25(1+\mathrm{f} /(3 \mathrm{mHz}))^{2} \mathrm{fm} / \mathrm{s}^{2} / \sqrt{\mathrm{Hz}}$ to the direct acceleration noise on the test masses [3]. Using this value, the overall acceleration of the RTM and NTM are provided in Tables 1 and 2, respectively. It is observed that residual accelerations of both test masses fall within the stated goals in the MBW.
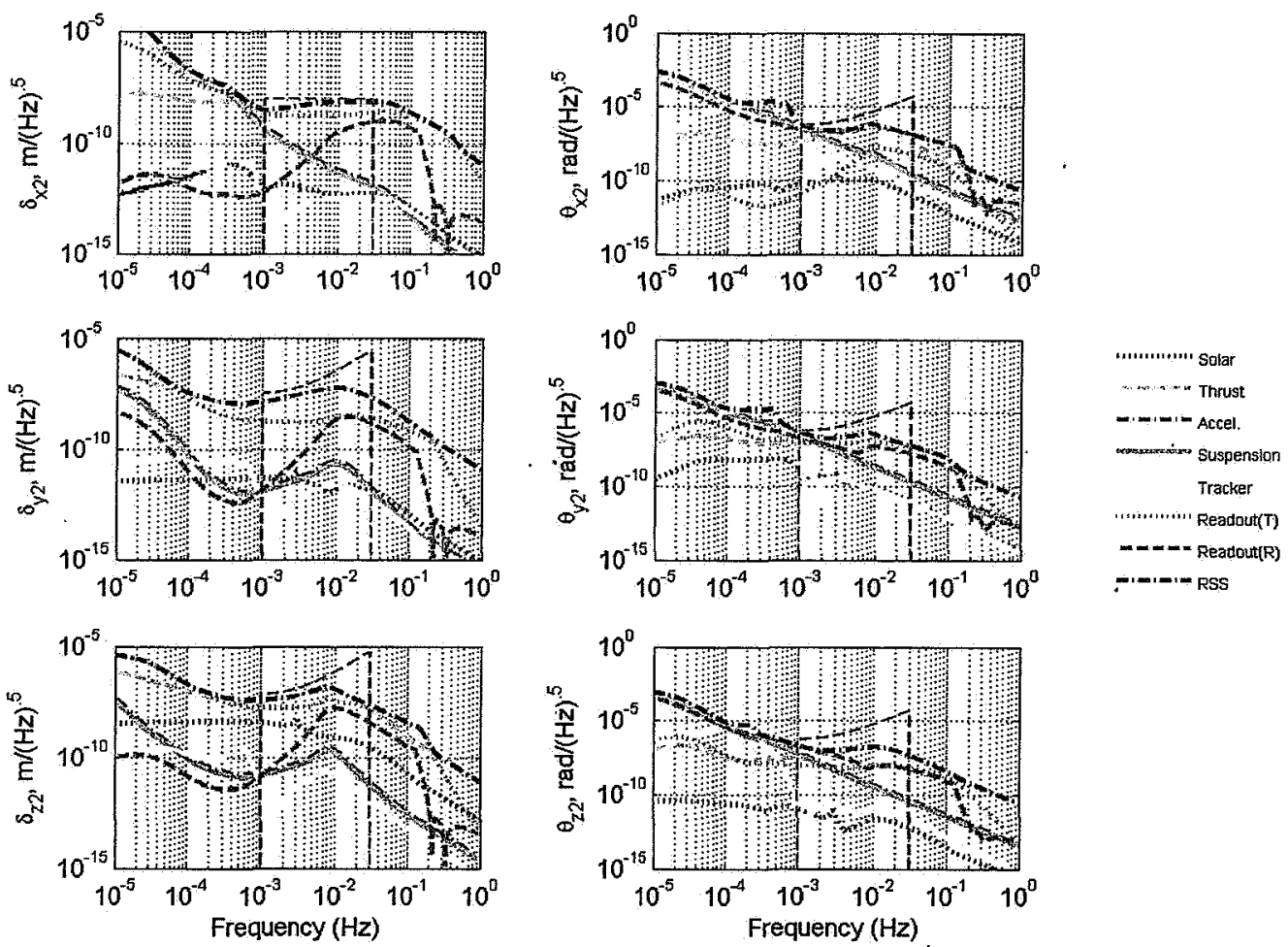

Figure 2 Root PSD Plots for the Non-Reference Test Mass

Table 1 Acceleration Performance of the Reference Test Mass

\begin{tabular}{|c|c|c|c|c|c|c|c|c|}
\hline & $1 \mathrm{mHz}$ & $2 \mathrm{mHz}$ & $3 \mathrm{mHz}$ & $5 \mathrm{mHz}$ & $8 \mathrm{mHz}$ & $10 \mathrm{mHz}$ & $20 \mathrm{mHz}$ & $30 \mathrm{mHz}$ \\
\hline $\begin{array}{c}\text { Residual } \\
\text { Acceleration, } \\
\text { Suspension (fm/s2) }\end{array}$ & 3.90 & 4.57 & 6.54 & 11.92 & 17.05 & 9.58 & 0.91 & 0.73 \\
\hline $\begin{array}{c}\text { Residual } \\
\text { Acceleration, } \\
\text { Stiffness (fm/s2) }\end{array}$ & 5.79 & 7.73 & 9.61 & 12.02 & 13.78 & 14.18 & 14.96 & 15.10 \\
\hline $\begin{array}{c}\text { Direct Forces } \\
\left(\mathrm{fm} / \mathrm{s}^{2}\right)\end{array}$ & 27.78 & 36.11 & 50.00 & 94.44 & 202.78 & 302.78 & 1136.1 & 25003 \\
\hline Total RSS (fm/s 2$)$ & 28.64 & 37.21 & 51.33 & 95.95 & 203.96 & 303.26 & 1136.2 & 25003 \\
\hline Requirements & 33.33 & 43.33 & 60.00 & 113.33 & 243.33 & 363.33 & 1363.3 & 30003 \\
\hline
\end{tabular}


Table 2 Acceleration Performance of the Non-Reference Test Mass

\begin{tabular}{|c|c|c|c|c|c|c|c|c|}
\hline & $1 \mathrm{mHz}$ & $2 \mathrm{mHz}$ & $3 \mathrm{mHz}$ & $5 \mathrm{mHz}$ & $8 \mathrm{mHz}$ & $10 \mathrm{mHz}$ & $20 \mathrm{mHz}$ & $30 \mathrm{mHz}$ \\
\hline $\begin{array}{c}\text { Residual } \\
\text { Acceleration, } \\
\text { Suspension (fm/s } 2)\end{array}$ & 9.35 & 15.07 & 14.14 & 32.33 & 49.01 & 51.67 & 51.30 & 51.08 \\
\hline $\begin{array}{c}\text { Residual } \\
\text { Acceleration, } \\
\text { Stiffness (fm/s } 2 \text { ) }\end{array}$ & 6.06 & 7.89 & 9.71 & 12.20 & 14.12 & 14.40 & 14.98 & 15.11 \\
\hline $\begin{array}{c}\text { Direct Forces } \\
\text { (fm/s } 2 \text { ) }\end{array}$ & 27.78 & 36.11 & 50.00 & 94.44 & 202.78 & 302.78 & 1136.1 & 25003 \\
\hline Total RSS (fm/s 2 ) & 29.93 & 39.92 & 52.86 & 100.56 & 209.1 & 307.49 & 1137.4 & 25003 \\
\hline Requirements & 33.33 & 43.33 & 60.00 & 113.33 & 243.33 & 363.33 & 1363.3 & 30003 \\
\hline
\end{tabular}

\section{CONCLUDING REMARKS}

Designs for the control modes of the ST7 Disturbance Reduction have been completed. There are six functional modes in the current control strategy. A high fidelity model of the system has been developed and was used to verify the performance of the control modes and the efficacy of the proposed transition strategy. Both time-domain and/or frequency-domain analyses indicate that the control modes do meet the ST7 requirements, particularly, those of the primary mission mode.

\section{REFERENCES}

1. Keiser, G. M., Buchman, S., Byer, R. L., Folkner, W. M., Hruby, V., and Gamero-Castaño, M., "Disturbance Reduction System for Testing Technology for Drag-Free Operation," SPIE Paper 4856-02, Astronomical Telescopes and Instrumentation Conference, Waikoloa, Hawaii, USA, August 2002.

2. Space Techlology 7, Dynamic Control System, Software Requirements Document, Doc. No. ST7-DRS-DCSFSW-REQ, Release 1.6, October, 2005.

3. LISA Pathfinder DFACS Requirements Specification, EADS Astrium, Doc. No. S2-ASD-RS-2001, Issue 1.4, July 18, 2005. 